\title{
Functional Organization of Human Supplementary Motor Cortex Studied by Electrical Stimulation
}

\author{
Itzhak Fried,' Amiram Katz,' Gregory McCarthy, ${ }^{1,2}$ Kimberlee J. Sass,, Peter Williamson,, ${ }^{1,2}$ Susan S. Spencer,' \\ and Dennis D. Spencer ${ }^{1}$
}

'Section of Neurosurgery and Department of Neurology, Yale University School of Medicine, New Haven, Connecticut 06510 and ${ }^{2}$ Neurology Service and Neuropsychology Laboratory, Veterans Administration Medical Center, West Haven, Connecticut 06516

\begin{abstract}
The presence of somatotopic organization in the human supplementary motor area (SMA) remains a controversial issue. In this study, subdural electrode grids were placed on the medial surface of the cerebral hemispheres in 13 patients with intractable epilepsy undergoing evaluation for surgical treatment. Electrical stimulation mapping with currents below the threshold of afterdischarges showed somatotopic organization of supplementary motor cortex with the lower extremities represented posteriorly, head and face most anteriorly, and the upper extremities between these two regions. Electrical stimulation often elicited synergistic and complex movements involving more than one joint. In transitional areas between neighboring somatotopic representations, stimulation evoked combined movements involving the body parts represented in these adjacent regions. Anterior to the supplementary motor representation of the face, vocalization and speech arrest or slowing of speech were evoked. Various sensations were elicited by electrical stimulation of SMA. In some cases a preliminary sensation of "urge" to perform a movement or anticipation that a movement was about to occur were evoked. Most responses were contralateral to the stimulated hemisphere. Ipsilateral and bilateral responses were elicited almost exclusively from the right (nondominant) hemisphere. These data suggest the presence of combined somatotopic organization and left-right specialization in human supplementary motor cortex.
\end{abstract}

The supplementary motor area (SMA) is part of Brodmann's area 6 and is situated on the mesial aspect of the cerebral hemisphere anterior to the primary motor representation of the foot. It is limited anteriorly by the prefrontal association cortex, posteriorly by Rolandic motor cortex (area 4), ventrally by the cingulate gyrus, and laterally by premotor cortex. Over a century ago, Munk (1881) and Horsley and Schafer (1888) demonstrated that electrical stimulation in this region in monkeys produced movements of the trunk, proximal upper extremity, and head. More modern experiments in animals (MacPherson et al., 1982a; Mitz and Wise, 1987) have shown that intracortical microstimu-

\footnotetext{
Received Apr. 29, 1991; revised June 20, 1991; accepted June 24, 1991.

This work was supported by NIH Grant NS06208.

Correspondence should be addressed to Itzhak Fried, M.D., Ph.D., Section of Neurosurgery, Yale University School of Medicine, 333 Cedar Street, New Haven, CT 06510.

Copyright (C) 1991 Society for Neuroscience $0270-6474 / 91 / 113656-11 \$ 05.00 / 0$
}

lation in this region evoked movements in the hindlimbs and forelimbs as well as orofacial, head, and eye movements. These movements are more complex than those evoked from primary motor cortex and often involve coactivation of several muscle groups (Wiesendanger et al., 1985). In humans, electrical stimulation of SMA has been shown to evoke complex motor synergies, assumption of postures, sensory responses, autonomic changes, vocalization, and speech arrest (e.g., Foerester, 1936; Brickner, 1940; Erickson and Woolsey, 1951; Penfield and Welch, 1951; Guidetti, 1957; Talairach and Bancaud, 1966; Van Buren and Fedio, 1976; Green et al., 1980).

The role of SMA in motor activity and its functional relationship with the primary motor and premotor cortices are unclear. According to one view, this area is situated high in a motor hierarchy and is involved in initiation and programming of movement (Eccles, 1982; Eccles and Robinson, 1984). This view of SMA as a "supramotor" area (Orgogozo and Larsen, 1979) is supported by electrophysiological studies showing unit activity in SMA prior to movement (Tanji and Kurata, 1982) and studies showing cerebral blood flow (CBF) changes in SMA with motor planning and in the absence of motor activity (Roland et al., 1980). According to another theory, SMA is a motor center that operates in parallel to primary motor cortex (Woolsey et al., 1952). Proponents of this view note that SMA has direct anatomical connections to downstream motor systems, especially to the spinal cord (Murray and Coulter, 1981; Macpherson et al., 1982b), and that changes in unit activity prior to movement are found not only in SMA but also in primary motor cortex (Tanji and Evarts, 1976; Kubota and Funashai 1982; Lucas et al., 1983).

Unlike the clearly somatotopic organization of primary motor cortex, the organization of supplementary motor cortex has been subject to controversy. Results vary among authors and by species. Woolsey et al. (1952) demonstrated somatotopic organization in the SMA of the macaque monkey in a rostral-to-caudal sequence with the head, forelimb, and hindlimb represented in that order. Although this finding is commensurate with anatomical and physiological studies (e.g., Brinkman and Porter, 1979; Murray and Coulter, 1981; Tanji and Kurata, 1982; Macpherson et al., 1982b), different results were obtained in the macaque by Penfield and Welch (1951) and Hughes and Mazurowski (1962). Subsequently, a more refined mapping of SMA was attempted by microstimulation methods. Although early studies yielded equivocal results (Smith, 1979; Palmer et al., 1981; Wise and Tanji, 1981; Macpherson et al., 1982a), more recent mi- 


\begin{tabular}{|c|c|c|c|c|c|c|c|}
\hline \multirow[b]{2}{*}{ Patient } & \multirow[b]{2}{*}{ Age } & \multirow[b]{2}{*}{ Sex } & \multirow[b]{2}{*}{ MRI abnormality } & \multicolumn{2}{|c|}{ Electrode array } & \multirow[b]{2}{*}{ EEG localization (ictal) } & \multirow[b]{2}{*}{ Tissue diagnosis } \\
\hline & & & & Left & Right & & \\
\hline 1 & 34 & $\mathbf{F}$ & Right SFG lesion & None & $2 \times 8$ & $\begin{array}{l}\text { Right parasagittal } \\
\text { peri-Rolandic }\end{array}$ & Cellular astrocytoma \\
\hline \multirow[t]{2}{*}{2} & 31 & $\mathbf{M}$ & None & $1 \times 8$ & $1 \times 8$ & Bioccipital & None \\
\hline & & & & $1 \times 8$ & $1 \times 8$ & & \\
\hline 3 & 33 & $\mathbf{M}$ & None & $2 \times 8$ & $1 \times 8$ & $\begin{array}{l}\text { Left frontal convexity } \\
\text { (premotor) }\end{array}$ & None \\
\hline 4 & 27 & $\mathrm{~F}$ & Left SFG lesion & $1 \times 8$ & $1 \times 8$ & $\begin{array}{l}\text { Left mesial frontal } \\
\text { (SMA) }\end{array}$ & Cortical hamartoma \\
\hline 5 & 22 & $\mathbf{M}$ & Left superior parietal lesion & $1 \times 8$ & $1 \times 8$ & Unlocalized & None \\
\hline 6 & 15 & $\mathbf{M}$ & None & $\begin{array}{l}1 \times 8 \\
1 \times 8\end{array}$ & $1 \times 8$ & $\begin{array}{l}\text { Left mesial frontal } \\
\text { (SMA) }\end{array}$ & No abnormality ${ }^{a}$ \\
\hline 7 & 34 & $\mathbf{F}$ & Right SFG lesion & None & $\begin{array}{l}1 \times 8 \\
1 \times 8\end{array}$ & Unlocalized & Cellular astrocytoma \\
\hline 8 & 31 & $\mathbf{F}$ & None & $2 \times 8$ & $1 \times 8$ & $\begin{array}{l}\text { Left frontal convexity } \\
\text { (premotor) }{ }^{a}\end{array}$ & Left SFG mild gliosis ${ }^{a}$ \\
\hline 9 & 21 & $\mathbf{M}$ & Right SFG lesion & $1 \times 10$ & $2 \times 16$ & Right frontal convexity & Glial hamartoma \\
\hline 10 & 31 & $\mathbf{M}$ & Absence right caudate & None & $2 \times 16$ & Unlocalized & $\begin{array}{l}\text { Right SFG gliosis and } \\
\text { reactive changes }\end{array}$ \\
\hline 11 & 35 & $\mathbf{M}$ & $\begin{array}{l}\text { Right cingulate lesion } \\
\text { Agenesis corpus callosum } \\
\text { (midbody) }\end{array}$ & $1 \times 8$ & $\begin{array}{l}1 \times 8 \\
1 \times 8\end{array}$ & Unlocalized & $\begin{array}{l}\text { Right cingulate fibrillary } \\
\text { astrocytic scar }\end{array}$ \\
\hline 12 & 30 & $\mathrm{~F}$ & None & $1 \times 8$ & $2 \times 16$ & Unlocalized & $\begin{array}{l}\text { Left SFG mild gliosis and } \\
\text { increased number of } \\
\text { neurons in molecular layer }{ }^{b}\end{array}$ \\
\hline 13 & 30 & $\mathrm{~F}$ & Left hippocampal atrophy & $1 \times 12$ & None & Left hippocampus & None \\
\hline
\end{tabular}

SFG, Superior frontal gyrus.

a Based on biopsy of area of ictal focus as determined by intracranial EEG recordings.

${ }^{b}$ Based on biopsy of area of maximal interictal activity as determined by intracranial EEG (no ictal localization).

crostimulation experiments by Gould et al. (1986) in the owl monkey and by Mitz and Wise (1987) in rhesus monkeys demonstrated rostrocaudal somatotopy in SMA with orofacial and eye movements most rostrally.

Demonstrating somatotopic organization in human SMA proved much more difficult than in animals. Penfield and Welch (1951) were unable to show somatotopic organization in SMA studied intraoperatively in patients with epilepsy undergoing craniotomy under local anesthesia. Using electrical stimulation in epileptic patients, Talairach and Bancaud (1966) suggested that "it is probable that the SMA admits a somatotopic organization: the face situated ahead of the upper limb; yet the responses at the level of the foot are difficult to prove, probably for technical reasons." The data reported in other studies (Van Buren and Fedio, 1976; Woolsey et al., 1979) are equivocal and insufficient. In addition, CBF studies (Orgogozo and Larsen, 1979) did not appear to support SMA somatotopy.

We now report a systematic study of human SMA using electric stimulation mapping in patients evaluated for epilepsy surgery. We investigated the medial aspect of the hemispheres through implanted subdural electrode arrays over a period of a few days, conducting an electrical stimulation protocol with adequate stimulus control. Using this method, we were able to address directly the question of somatotopic organization and to study in detail the responses obtained by electrical stimulation of the SMA.

\section{Materials and Methods}

Electrical stimulation mapping of mesial frontal cortex was carried out in 13 patients with intractable seizures as part of their evaluation for surgery (Table 1). All patients except two (patients 6 and 9, Table 1) were right handed, and all except one (patient 6) were found to have left hemisphere dominance for speech by the intracarotid amobarbital test. Five had lesions at or near the medial aspect of the frontal lobe detected by magnetic resonance imaging (MRI). These patients were studied in order to determine the relation of the lesion and areas of seizure onset to critical functional areas. The other eight patients had no apparent medial lesions but, in the absence of sufficient localizing EEG evidence, underwent invasive monitoring that included the medial cortical structures. One of these patients (patient 5, Table 1) was later found in a subsequent MRI scan to have a small lesion in the left superior parietal lobule. The locations of the lesions as well as the results of intracranial seizure monitoring are described in Table 1 .

All patients underwent craniotomy under general anesthesia. The dura was opened laterally and reflected toward the midline until the medial aspect of the hemisphere, and the falx cerebri were visualized. One or two flexible subdural strips (made by Ad-Tech Medical Instrument Corporation, Racine, WI), consisting of one or two rows of 8-16 stainless-steel circular contacts, were inserted subdurally to cover the mesial aspect of the hemisphere. The exposed surface of the contacts was of $2.4 \mathrm{~mm}$ diameter and the distance between adjacent contacts (center to center) ranged from 0.5 to $1.0 \mathrm{~cm}$. In 9 of the 12 patients, an additional subdural strip was inserted through an opening in the falx cerebri to cover the mesial aspect of the opposite hemisphere. The area of frontoparietal cortex covered by the stimulation array varied among patients according to the size of the array used (Table 1). The anterior-posterior (AP) extent of coverage varied between 8 and $16 \mathrm{~cm}$. 
Table 2. Distribution of sites where stimulation evoked a response $(n=129)$ according to type of response, complexity of response, and laterality of response

\begin{tabular}{lccc} 
& $\begin{array}{l}\text { Left } \\
\text { stimulation }\end{array}$ & $\begin{array}{l}\text { Right } \\
\text { stimulation }\end{array}$ & Total \\
\hline Type of response & 40 & 41 & 81 \\
Motor & 17 & 22 & 39 \\
Subjective-sensory & 3 & 0 & 3 \\
Vocalization & 10 & 5 & 15 \\
Speech arrest/slowing & & & \\
Complexity of response (motor or sensory) & 21 & 19 & 40 \\
Simple & 25 & 24 & 49 \\
Regional & 9 & 19 & 28 \\
Complex & & & \\
Laterality of response & & & \\
(1) Excluding facial and occular responses & 36 & 81 \\
$\quad$ Contralateral & 45 & 6 & 6 \\
$\quad$ Ipsilateral & 0 & $13^{a}$ & 14 \\
$\quad$ Bilateral & 1 & & \\
(2) Facial and ocular responses & & 6 & 11 \\
$\quad$ Contralateral & 5 & 1 & 1 \\
Ipsilateral & 0 & 1 & 4 \\
$\quad$ Bilateral & $3 b$ & & \\
\hline
\end{tabular}

${ }^{a}$ Two responses involved bilateral neck and chest sensations near the midline.

${ }^{b}$ Two responses of bilateral eye blinking.

Postoperatively, patients underwent EEG monitoring and electrical stimulation mapping over a period of several days. In nine patients, bilateral mesial stimulation was carried out. Four patients underwent unilateral stimulation mapping: three had right mesial electrode strips and one had a left mesial strip.

Electrical stimulation was delivered in a bipolar mode through adjacent contacts by a Grass $\mathbf{S} 88$ stimulator, while the patient was naming objects depicted on flash cards. Stimulation consisted of $100 \mu \mathrm{sec}$ monophasic square wave pulses delivered at $50 \mathrm{~Hz}$ at a constant current for a duration of $5 \mathrm{sec}$. If an overt response occurred, stimulation would be terminated prior to the $5 \mathrm{sec}$ limit. Stimulation was applied in increasing increments of $0.5 \mathrm{~mA}$ or $1 \mathrm{~mA}$. Once an overt behavioral response or an electric afterdischarge was obtained with stimulation at a certain site, it would usually be excluded from stimulation at higher currents. The maximum current utilized was $10 \mathrm{~mA}$. Only those responses not associated with afterdischarges were considered for functional localization.

\section{Results}

Altogether, 299 sites over the mesial hemispheric surface were electrically stimulated in 13 patients. Responses were obtained with stimulation of 129 sites.

\section{Motor responses}

The majority of the responses were overt movements and were elicited at 81 sites $(62.8 \%$ of all sites where responses were evoked; Table 2). We classified these into three categories: simple, regional, and complex responses. Simple responses were discrete movements involving one joint or restricted to the digits of one extremity. Regional responses involved several joints, but were confined to one extremity or to a region, that is, face, neck, trunk, one upper extremity, or one lower extremity. Orofacial responses were classified as simple only if they clearly involved a single muscle group. Otherwise, they were classified as regional. Responses that involved several body regions were
Table 3. Participation of various body regions in the motor and sensory responses (speech-related responses excluded) evoked by electrical stimulation $(n=117 \text { sites })^{a}$

\begin{tabular}{lc}
\hline Lower extremities & $51(43.6 \%)$ \\
Upper extremities & $68(58.1 \%)$ \\
Trunk & $5(4.3 \%)$ \\
Neck & $5(4.3 \%)$ \\
Face & $10(8.6 \%)$ \\
Eyes & $6(5.1 \%)$
\end{tabular}

a Note that at some sites complex responses involving several body regions were elicited.

classified as complex. A wide spectrum of responses is illustrated by stimulation results in patients 2 (Fig. 1) and 8 (Fig. 2).

The temporal profile of the movements evoked varied. Some movements evolved rapidly while others developed slowly, often resulting in the assumption of a posture. These relatively slow movements were often accompanied by a tremor (e.g., see site 5-6, Fig. 4). There appeared to be a relationship between the complexity of the response and its temporal profile. The simple movements were commonly fast, whereas the majority of the regional and complex movements developed more slowly. Some of the responses were repetitive, usually involving a regional response with one extremity, repeated several times until stimulation was terminated.

\section{Subjective-sensory responses}

In addition to the overt motor responses, but less frequently, electrical stimulation elicited reports of a variety of sensory experiences. These were of three types: (1) sensation of tingling, numbness, or very rarely a sensation of warmth or mild pain (18 sites in seven patients); (2) subjective experience of movement in the absence of overt motor activity (six sites in three patients) (e.g., one patient reported "I feel my arm is moving"; however, no arm movement was observed); and (3) a subjective "urge" to perform a movement or anticipation that a movement was going to occur ( 15 sites in four patients). Figure 3 illustrates stimulation results in a patient with several of these "urge" responses. Note that at some sites where such responses were elicited, stimulation at a higher current evoked an overt motor response (A3-A4, Fig. 3), but when a movement was elicited by a higher current it would not always be the same one for which an "urge" had been experienced at a lower stimulus intensity. Often, the overt movement would be more proximal (A4-B4, Fig. 3), and rarely it would involve a different limb (A3-B3, Fig. 3). At some sites where an "urge" to perform a movement had been elicited, no overt movements were evoked when the stimulation current was raised.

The sensory responses were also of varying degrees of topographic complexity and thus could be classified in a similar fashion to the motor responses. Simple sensory responses involved a discrete sensation limited to the space between two adjacent joints or around one joint, while regional responses involved one limb or body region. Complex sensory responses involved several body regions.

The subjective-sensory responses were elicited at 39 sites in nine patients $(30.2 \%$ of the sites where responses were evoked; see Table 2). At three of these sites, motor responses were obtained at the same time. 


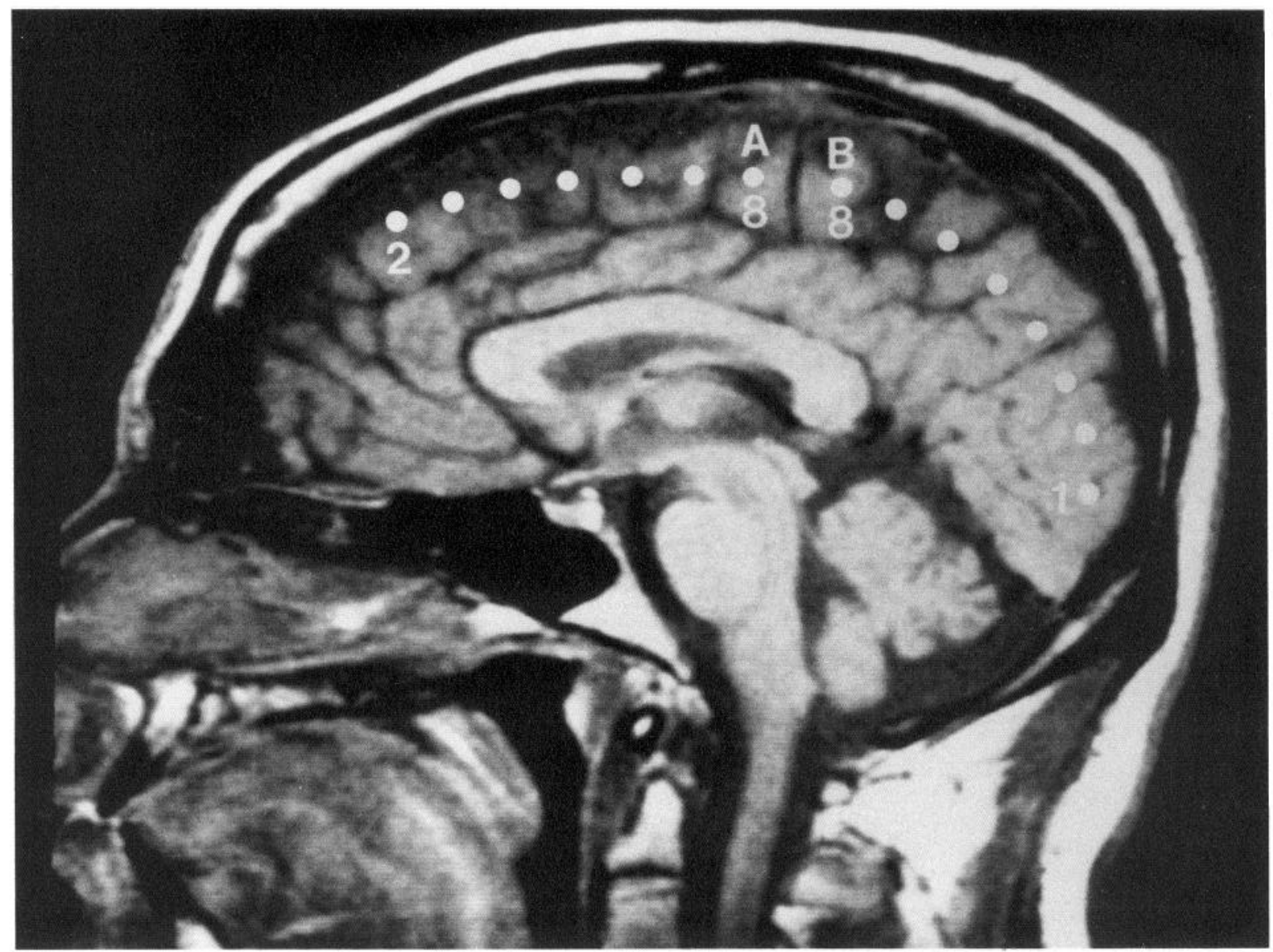

Figure 1. Schematic view of two $1 \times 8$ subdural electrode arrays (anterior array $A$ and posterior array $B$ ) placed on the mesial aspect of the left hemisphere in patient 2. Distance between adjacent contacts is $1 \mathrm{~cm}$. The drawing of the array was based on intraoperative evaluation of strip location. Contacts were drawn on a left sagittal MRI obtained preoperatively. Following are the results of electrical stimulation between adjacent contact points at the minimal current (in $\mathrm{mA}$ ) that yielded a response without evidence of afterdischarge. Proceeding along the strips in a posteriorto-anterior direction, note progression from responses involving distal lower extremity to responses involving proximal lower extremity and pelvis and then to a response involving upper extremity. Most anterior are responses related to vocalization and speech. Responses at B6-B7 and B7-B8 were sudden and brief and were elicited at low stimulation currents. These sites could be part of primary motor cortex.

B6-B7 (2.5 mA) quick right hip flexion and ankle dorsiflexion

B7-B8 (4 mA) quick right hip flexion and ankle dorsiflexion

A7-A8 (8 mA) right hip and knee flexion, pelvic thrust

A6-A7 (10 mA) right forearm elevation

\section{Complexity}

Altogether, $34.2 \%$ of the motor and sensory responses were simple, $41.9 \%$ were regional, and $23.9 \%$ were complex (Table 2 ). The distribution of the responses according to body regions is described in Table 3. The upper extremities were represented in $58.1 \%$ of the responses and the lower extremities in $43.6 \%$. Some of these responses included both upper and lower extremities; that is, they were complex responses. Because the medial aspect of the hemisphere just behind the traditionally delineated SMA includes Rolandic cortex, it is possible that some of the simple or regional responses involving the lower extremities were actually obtained by stimulation of primary motor or somatosensory cortices. Only 12 such responses were elicited in this study, and these were usually sudden, quick, and brief (e.g., sites B6-B7 and B7-B8 in Fig. 1).

Responses involving neck and trunk were relatively rare and usually occurred in the context of complex synergies. In one patient (patient 11), some of the contacts were on the surface

$\begin{array}{lll}\text { A5-A6 } & (4 \mathrm{~mA}) & \begin{array}{l}\text { speech arrest for naming but could count } \\ \text { continuous vocalization of vowel }\end{array} \\ \text { A4-A5 } & (6 \mathrm{~mA}) & \begin{array}{l}\text { continuous vocalization of vowel } \\ \text { complete speech arrest }\end{array}\end{array}$

of the cingulate gyrus. Stimulation at two of these sites in the right cingulate cortex evoked numbness at the chest and the posterior aspect of the neck near the midline.

At 16 sites, facial responses were elicited, and six of these involved the eyes. At three of those sites, conjugate eye movements to the contralateral side were evoked. In patient 11 , stimulation of two sites on the posterior part left cingulate gyrus evoked forced rapid eye blinking.

\section{Speech-related responses}

In addition to the motor and sensory responses, electrical stimulation elicited speech-related responses. These were of three types: (1) vocalization, (2) speech arrest, and (3) speech slowing or hesitancy. Vocalization was elicited with stimulation of three sites in two patients (e.g., Fig. 1, sites A4-A5, A5-A6). Both patients had bilateral electrode placement, but vocalization was evoked only with stimulation of the left (dominant) hemisphere. Vocalization was characterized by a continuous vowel sound emitted for the duration of stimulation. Clear speech arrest was 


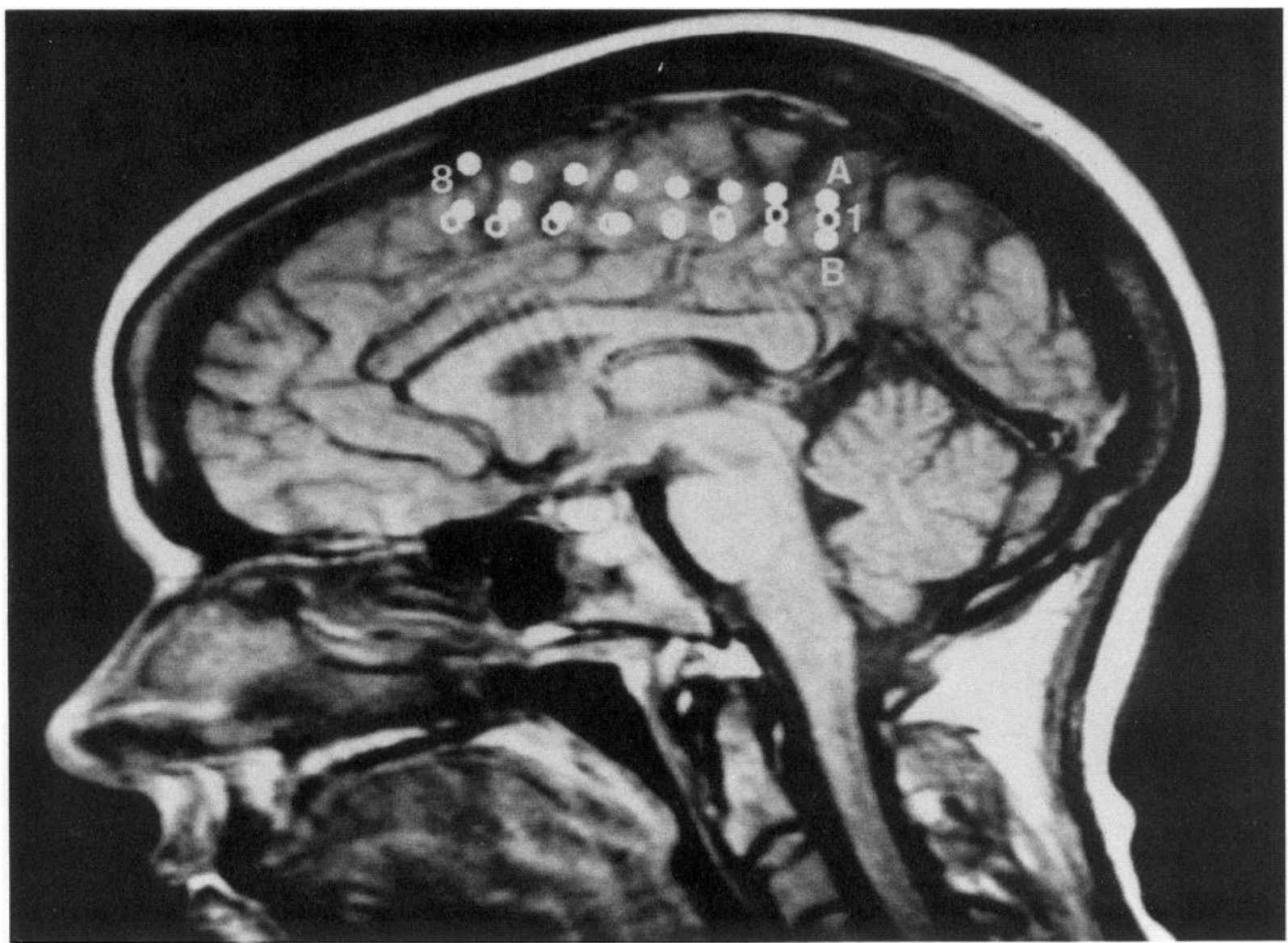

Figure 2. Reconstruction of subdural electrode arrays placed on the mesial aspect of each hemisphere in patient 8 . On the mesial aspect of the left hemisphere a $2 \times 8$ contact array (rows $A$ and $B$ ) was placed. On the mesial aspect of the right hemisphere an eight-contact strip was placed (open circles). The distance between adjacent contacts was $1 \mathrm{~cm}$. Reconstruction of the electrode arrays is based on postoperative skull radiograms superimposed on preoperative MRIs of the brain. Following are the positive responses obtained from stimulation between adjacent contact points at the lowest current that yielded a response without evidence of afterdischarge. Note that stimulation of all the right hemispheric sites elicited bilateral responses whereas stimulation of only one left hemispheric site evoked bilateral response. Note also the presence of a general pattern of somatotopic organization with facial and speech-related responses most anteriorly. Behind these areas is a relatively large region of upper extremity responses.

\section{Right}

2-3 Bilateral shoulder external rotation and elbow extension

3-4 Bilateral arm abduction and elbow flexion

3-4 Bilateral arm abduction and elbow flexion

4-5 Bilateral arm tingling, left elbow flexion, tongue deviation to left

5-6 Bilateral perioral cheek tingling (at $0.5 \mathrm{~mA}$ higher also left conjugation eye deviation

\section{Left}

A2-A3 Tingling right foot, spreading to right leg and arm

B2-B3 Bilateral forearm and hand sensation, right greater than left

evoked at 10 sites (six left hemispheric and four right hemispheric) in six patients (see examples in Figs. 1, 2). At three of these sites, orofacial movements were elicited at the same time, and at two sites upper extremity movement was triggered along with speech arrest (e.g., Fig. 2, site B6-B7). At one site, speech arrest was elicited during a naming task, but stimulation did not disrupt counting (Fig. 1, A5-A6). Speech slowing or hesitancy was evoked with stimulation of four sites (three left hemispheric and one right hemispheric) in two patients. At one of these sites, an "urge" to move the upper extremity was elicited along with speech slowing.

$\begin{array}{ll}\text { A3-A4 } & \begin{array}{l}\text { Tingling right leg, abduction right forearm, flexion right } \\ \text { elbow }\end{array} \\ \text { B3-B4 } & \text { Right arm } \text { abduction } \\ \text { A4-A5 } & \text { Right elbow flexion } \\ \text { B4-B5 } & \text { Tingling } \text { right small finger } \\ \text { A5-A6 } & \text { Right } \text { wrist } \text { extension } \\ \text { B5-B6 } & \text { Right elbow flexion and grasping movement with right } \\ & \text { hand } \\ \text { A6-A7 } & \text { Speech arrest } \\ \text { B6-B7 } & \text { Speech arrest } \text { and right hand finger flexion } \\ \text { A7-A8 } & \text { Speech arrest } \\ \text { B7-B8 } & \text { Speech arrest }\end{array}$

\section{Somatotopic organization}

A pattern of somatotopic organization emerged from electrical stimulation in our patients. Stimulation at the most posterior region of the SMA yielded responses involving the distal part of the lower extremity; these may be difficult to distinguish from the primary motor or sensory responses elicited in the leg area of Rolandic cortex. As the stimulation was applied more anteriorly, responses from proximal lower extremity, trunk, proximal upper extremity, distal upper extremity, neck, and finally face were recruited, in that order (Fig. 1). Within the "face 


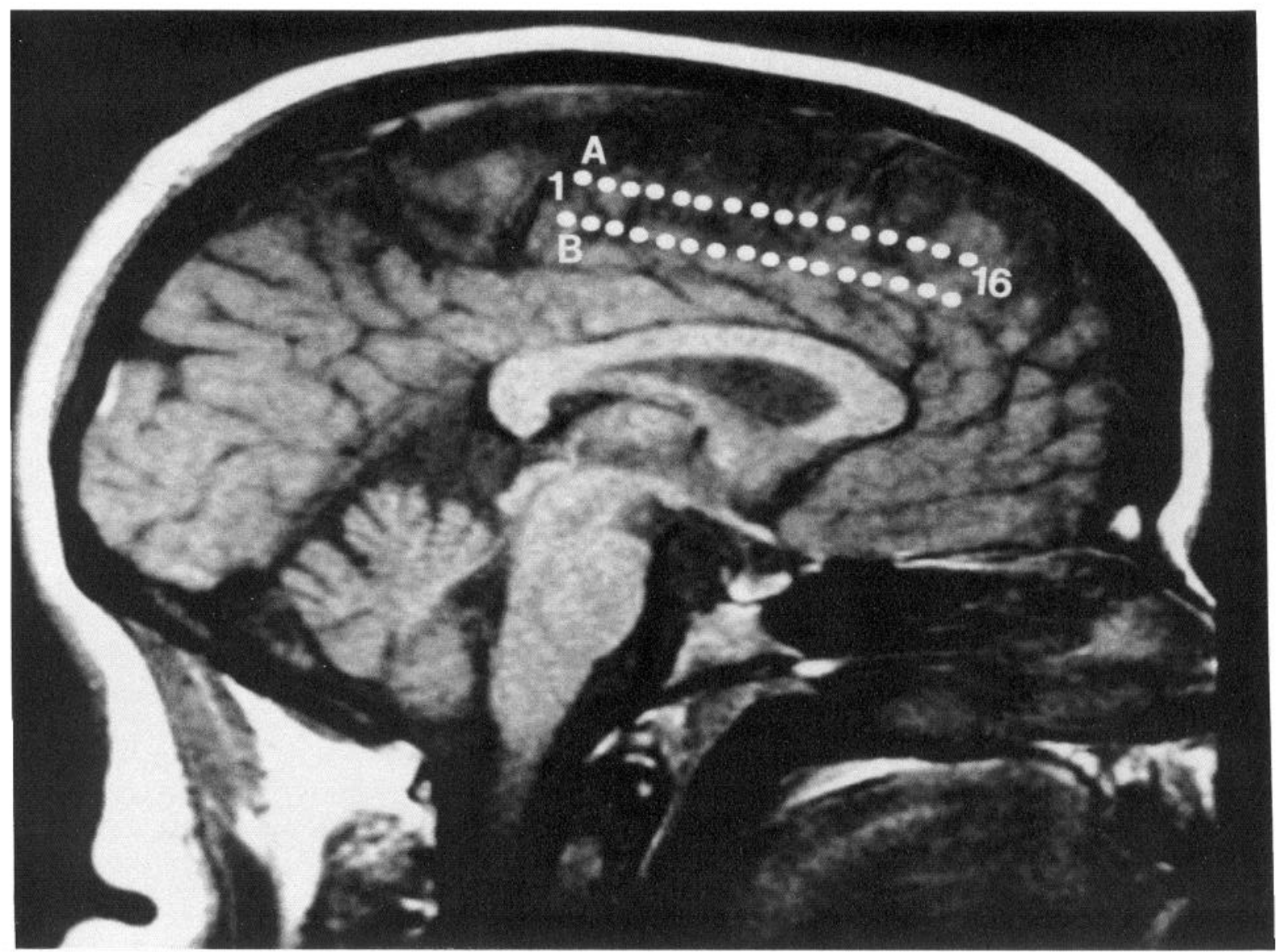

Figure 3. Reconstruction of a $2 \times 16$ subdural electrode array (rows $A$ and $B$ ) placed on the mesial aspect of the left hemisphere in patient 12 , based on postoperative skull radiograms of the array that were superimposed on the preoperative MRI of the brain. Distance between adjacent contacts is $0.5 \mathrm{~cm}$. Following are the results of electrical stimulation between adjacent contact points at currents (in $\mathrm{mA}$ ) below the threshold for afterdischarges. Note the sites where stimulation elicited an "urge" to perform a movement.

\begin{tabular}{|c|c|c|}
\hline A1-B1 & $(5 \mathrm{~mA})$ & $\begin{array}{l}\text { Right foot inversion followed by knee flexion } \\
\text { (repetitive) }\end{array}$ \\
\hline A1-A2 & $(8 \mathrm{~mA})$ & Right foot inversion \\
\hline B1-B2 & $(5 \mathrm{~mA})$ & Right knee flexion \\
\hline A2-B2 & $(4 \mathrm{~mA})$ & Right hip adduction, knee flexion \\
\hline A2-A3 & $(6 \mathrm{~mA})$ & Trunk rotation to the left \\
\hline 2-B3 & $(4 \mathrm{~mA})$ & Right hip adduction, knee flexion \\
\hline A3-B3 & $(4 \mathrm{~mA})$ & "Urge to move right leg inward" \\
\hline \multirow[t]{2}{*}{ A3-A4 } & $(4 \mathrm{~mA})$ & $\begin{array}{l}\text { Feeling a "need to do something with right } \\
\text { hand" }\end{array}$ \\
\hline & $(5 \mathrm{~mA})$ & $\begin{array}{l}\text { Right hand pronation, elbow extension, } \\
\text { shoulder abduction ("push away") }\end{array}$ \\
\hline \multirow[t]{3}{*}{$\begin{array}{l}\text { B3-B4 } \\
\text { A4-B4 }\end{array}$} & $\begin{array}{l}(9 \mathrm{~mA}) \\
(5 \mathrm{~mA})\end{array}$ & $\begin{array}{l}\text { Neck extension, rotation of head to right } \\
\text { "Urge to move right thumb and index finger" }\end{array}$ \\
\hline & $(5.5 \mathrm{~mA})$ & $\begin{array}{l}\text { Slight movement right hand followed by } \\
\text { shoulder internal rotation }\end{array}$ \\
\hline & $(6 \mathrm{~mA})$ & Right shoulder internal rotation \\
\hline \multirow[t]{2}{*}{ A4-A5 } & & $\begin{array}{l}\text { "Urge to move right arm," slight extension } \\
\text { right elbow. }\end{array}$ \\
\hline & $(5 \mathrm{~mA})$ & $\begin{array}{l}\text { Right elbow extension, right forearm prona- } \\
\text { tion, flexion of fingers ("pick up" motion) }\end{array}$ \\
\hline & & Head rotation to right \\
\hline & & $\begin{array}{l}\text { Feeling as if right hand movement "was } \\
\text { about to occur" }\end{array}$ \\
\hline
\end{tabular}

region" are also sites where stimulation elicited eye movements. Most anteriorly, stimulation elicited responses related to speech: vocalization, speech arrest, or slowing or hesitation in speech or naming. In general, the complex responses conformed to the

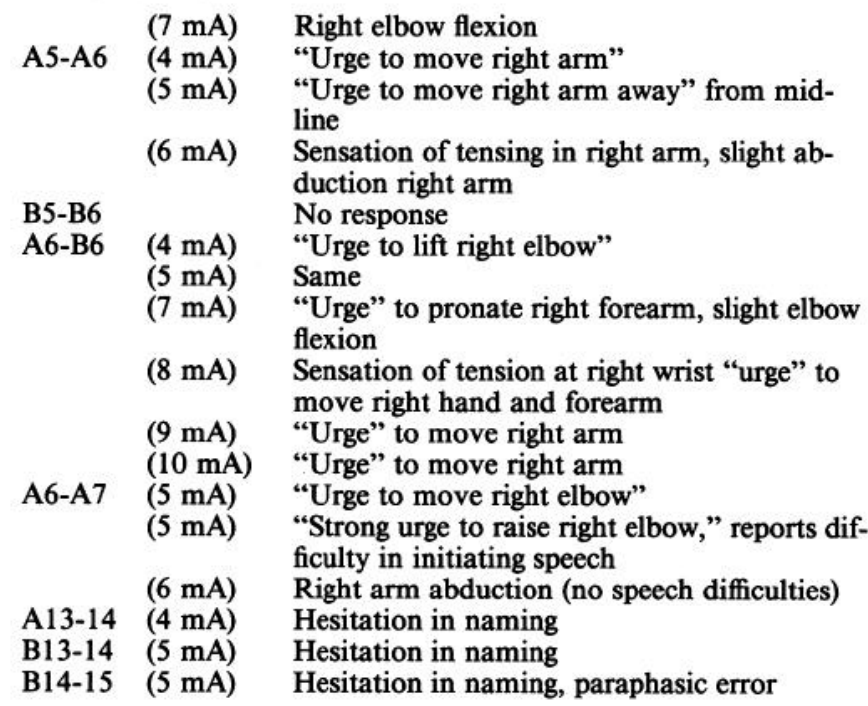

somatotopic organization. For example (Fig. 2), a complex response involving the right leg and forearm is evoked at a site (A3-A4) that is posterior to a site where stimulation evoked right finger flexion and speech arrest (B6-B7). In transitional 


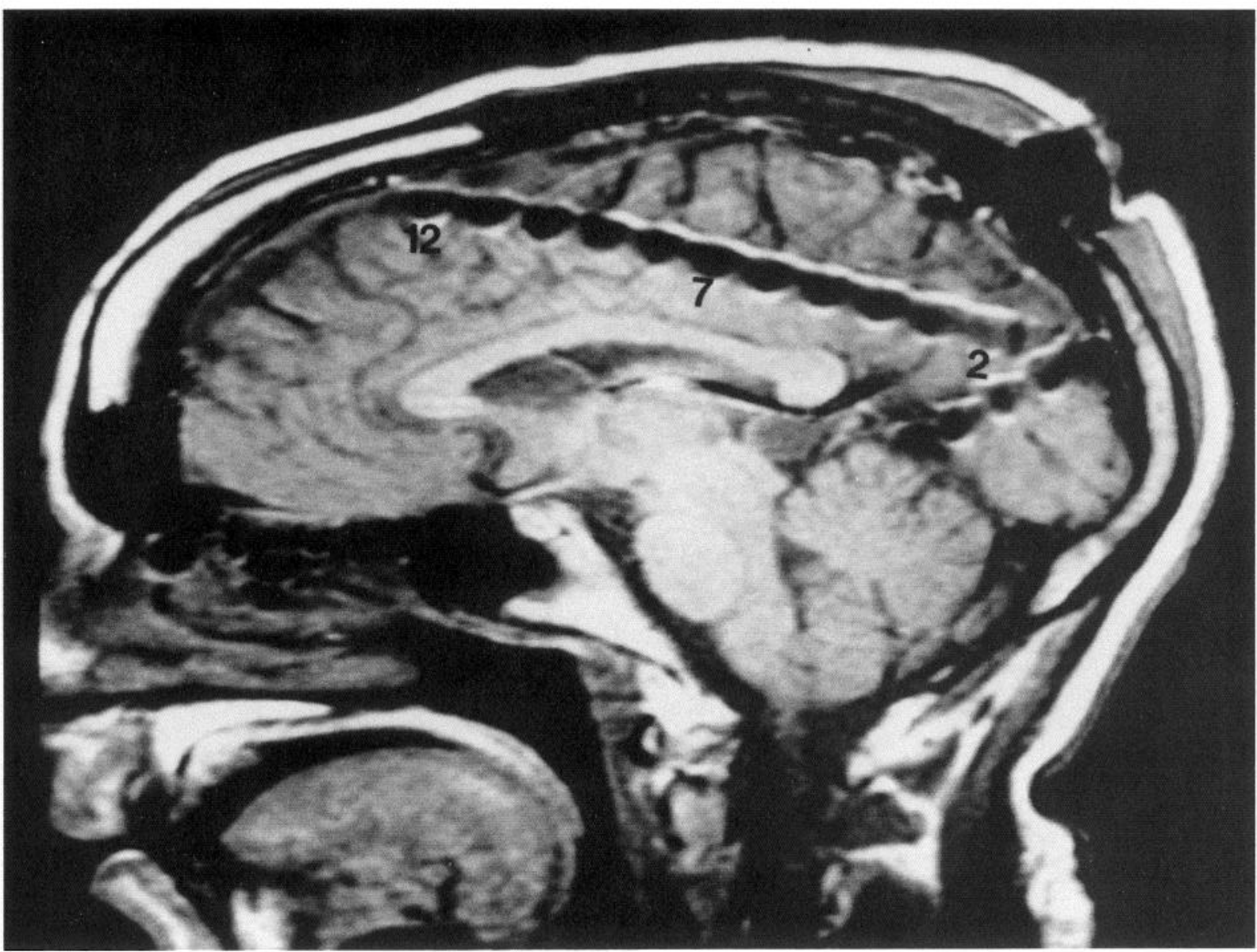

Figure 4. Sagittal MRI of a 12 contact subdural electrode strip over the mesial surface of the left hemisphere in patient 13. MRI was obtained postoperatively with the electrodes in situ, resulting in a minor hypodense circular artifact around each contact. The distance between adjacent contacts was $1 \mathrm{~cm}$. Following are the responses obtained by electrical stimulation between adjacent contact points at currents below the threshold of afterdischarges. Note the progression of the responses along the right upper extremity from shoulder and arm to forearm and hand as the stimulation is advanced from posterior to anterior sites.

5-6 (5 mA) Sensation of tensing of upper right arm as if patient "was going to move it"

(5.5 mA) Tremor of right arm above elbow

6-7 (4 mA) External rotation of right shoulder

7-8 (3.5 mA) Internal rotation of right shoulder, tremor right arm and forearm

8-9 (3.5 mA) Right elbow flexion and grasping with right hand

areas between neighboring regional somatotopic representations, stimulation often elicited complex movements involving the body regions represented in these adjacent regions (Figs. 1, 2). Within an area of representation of a limb, there was often a finer organization. For instance, distal upper extremity responses were elicited at sites anterior to those where proximal upper extremity responses were evoked (Fig. 4).

In order to test the hypothesis that there is somatotopic organization in SMA, for each patient the sites with stimulationevoked responses in each hemisphere were rank ordered according to two variables (Fig. 5). The first variable was the AP location of the site along the mesial surface of the hemisphere. In this ranking scheme, the most posterior site was assigned the lowest number and the most anterior site the highest number. The second variable was the rostral-caudal (RC) body location of the response elicited by stimulation at the site. Here, the numerical order of the ranking increased with the rostral extent of the response, in the same order as in primary motor cortex somatotopy, that is, lower extremity (from distal to proximal), trunk, upper extremity (from proximal to distal), neck, and face. The highest numerical rank was assigned to sites with speechrelated responses (i.e., in front of the "face region"). In this fashion, each of the 129 sites with stimulation-evoked response was assigned two numerical ranks, one according to the AP location in SMA of the stimulation site, and the other according to the RC location in the body of the response elicited by electrical stimulation at that cortical site. Both ranks were relative to the sites within that individual SMA and not with respect to sites in other patients. Spearman's coefficient of correlation between these two variables, stimulation location and response location, for the group of 129 sites with stimulation-evoked responses was 0.744 ( $p<0.001 ; n=129$; Fig. 5).

\section{Laterality of responses}

Most of the responses elicited were contralateral to the side of stimulation (Table 2). Bilateral responses or responses ipsilateral to the stimulated hemisphere were evoked only at 25 sites in seven patients. Five of these were facial (including blinking), 
two involved chest and neck, and 18 involved the extremities. Compared to the extremities, a higher proportion of facial responses were bilateral. Interestingly, all ipsilateral and bilateral extremity responses except one were elicited from stimulation of the right (nondominant) hemisphere (Table 2). However, two of the seven patients with bilateral and ipsilateral responses had only right mesial electrode strips.

\section{Discussion}

Interpretation of human electrical stimulation data poses some problems, mainly with regard to stimulus control and range. One point of criticism of data obtained from human SMA stimulation is that the complex synergies evoked by surface stimulation might represent current spread. In our patients, neighboring sites were monitored and the responses considered valid for localization only when they were not accompanied by afterdischarges. Even with such attention to stimulus control, about two-thirds of the responses involved several muscle groups and sometimes more than one extremity or body region. This finding is consistent with animal microstimulation studies that suggest that motor responses evoked by stimulation of SMA represent more divergent efferent zones and are more complex than those obtained by stimulation of primary motor cortex. Mitz and Wise (1987), using electrical stimulation of the rhesus monkey cortex at ranges up to only $65 \mu \mathrm{A}$, obtained "noncontiguous" responses (i.e., coactivation of several noncontiguous joints) in $9-16 \%$ of the sites. At $19-35 \%$ of the sites, several joints (albeit contiguous) were activated. Wiesendanger et al. (1985), when microstimulating monkey SMA at threshold levels (as low as 30 $\mu \mathrm{A}$ ), found EMG evidence of coactivation of combinations of muscles such as hand extensors, triceps, and brachioradialis or deltoid, brachioradialis, pectoralis, and triceps.

Previous human studies demonstrated a high incidence of complex motor responses evoked by stimulation of SMA. Foerster (1936) stimulated the medial aspect of area 6 and produced adversive movements of the head, eyes, and trunk, and flexor and extensor synergies of the contralateral arm and leg. Penfield and Welch (1951) obtained motor responses from stimulation of SMA in their patients. They divided these into the following categories: assumption of postures, maneuvers (e.g., stepping), and rapid incoordinate movements. Similar to their findings, most of the responses that we observed were of the first type, that is, assumption of postures involving one or more body regions, and therefore by our definition regional or complex responses. A close look at the Penfield and Welch data reveals that most of the motor responses that they observed would have been classified in our terminology as complex. For instance, 26 responses involving arm movements were observed in 10 patients, and in all but 8 responses the arm movement was part of a complex response involving leg movement, inability to speak, eye responses, or head movement. In our study, complex responses were elicited in $25 \%$ of sites with stimulation-evoked responses. The lower incidence of complex responses in our patients may be explained by more attention to control of stimulation current spread.

Of particular interest were the observations in our patients who reported perception of ongoing movement, anticipation of movement, or the "urge" to perform a movement, all in the absence of overt motor activity. We know of no previous reports of such responses, a fact that can probably be ascribed to the difficulty in observing them. First, good stimulus control is necessary since these responses are often obtained only at threshold

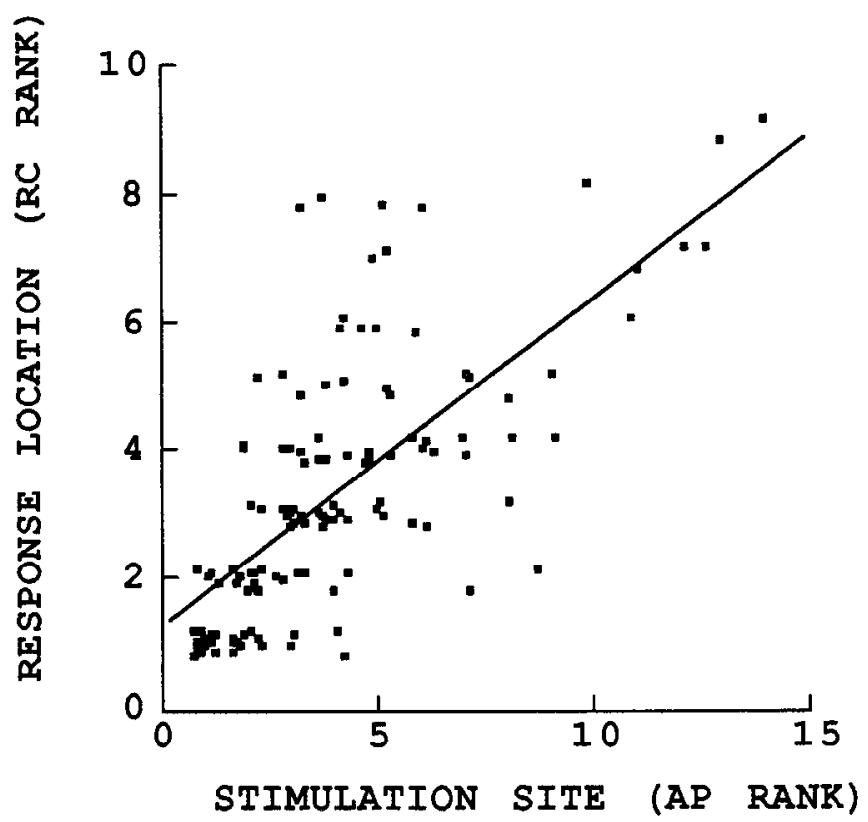

Figure 5. The distribution of trials with stimulation-evoked responses according to two variables: (1) the AP location of the site of stimulation over the mesial surface of the hemisphere and (2) the RC location of response on the body. Each variable is expressed nonparametrically in integers representing rank ordering within a stimulation array in each patient. For the variable describing location of stimulation sites, a greater numerical rank represents a more anterior location on the mesial aspect of the hemisphere. For the variable describing body location of the response, a greater numerical rank represents a more rostral location on the body. Spearman's coefficient of correlation between these two variables would be 1.00 for a perfect somatotopic map. The computed coefficient in our study was $0.744(p<0.001 ; n=129)$.

currents above which overt motor activity would be readily elicited. Also, the patients may not be inclined to report such experiences in paradigms where gross motor activities and more objcctive results are cxpected, cspecially if the stimulation is carried out intraoperatively. The observations of such subjective responses are obviously unique to human studies but may offer an insight into the function of SMA. It has been suggested that SMA participates in the programming and initiation of movement (Eccles, 1982). CBF studies claim changes in SMA regions associated with programming of a sequence of movements without actually executing it (Roland et al., 1980). Neurophysiological studies show unit activity (e.g., Tanji and Kurata, 1982) and slow potentials (Deecke, 1985, 1987) in SMA prior to execution of motor activity. Previous investigators described alteration of the ability to initiate or continue voluntary activity with stimulation of SMA (Penfield and Welch, 1951) or with lesions of SMA (LaPlane et al., 1977; Freund, 1985; Freund and Hummeisheim, 1985). Resection of the SMA usually causes a substantial reduction in spontaneous motor activity more pronounced on the side of the body contralatcral to the lesion, and often a severe reduction in speech output. Interestingly, these deficits are transient and usually resolve within a few weeks (LaPlane et al., 1977).

Previous studies have demonstrated sensory responses obtained by electrical stimulation of human SMA, but these were mostly poorly localized sensations referred to wide areas of the body (Penfield and Welch, 1951; Van Buren and Fedio, 1976). In our study, sensory responses of varying topographic com- 
plexity were evoked, from simple responses involving a discrete focal sensation (numbness or tingling) to complex sensations involving various regions of the body. Similar to the findings by Van Buren and Fedio (1976), we found a higher incidence of sensory responses compared to the data by Penfield and Welch (1951). Again, this is best explained by attention to stimulation control and current spread. Sensory responses are more easily missed at threshold currents, and once the current is raised too much, a motor response is evoked.

In this study, vocalization was obtained only by stimulation of left (dominant) SMA sites. This occurred in two patients, and both underwent right SMA stimulation as well, but without resultant vocalization. Penfield and Welch (1951) reported vocalization with stimulation of right (nondominant) SMA as well as left SMA. Morris et al. (1988) stimulated the right SMA in three patients. In one, stimulation evoked vocalization (no stimulation of left SMA was performed). Talairach and Bancaud (1966) elicited vocalization from mesial sites in 26 patients and found no left-right differences in the distribution of those sites, but Chauvel et al. (1985) later stated that "the probability of eliciting vocalization was significantly higher on stimulation of the dominant hemisphere." Penfield and Welch (1951) reported that the most characteristic vocalization response was "rhythmic, or intermittent sound, but instantaneous exclamation, continuous prolongation of a vowel sound, and the repetition of a word or of a meaningless syllable, or combination of syllables, have been observed as well." We have observed only continuous prolongation of a vowel sound, occasionally with some modulation of pitch and volume. Because of the small number of vocalization sites in the present study, it is impossible to draw any conclusions as to the cerebral lateralization of this phenomenon.

Consistent with other reports, speech arrest or speech slowing were evoked by stimulation of either left or right SMA in our patients. Penfield and Welch (1951) obtained speech arrest and slowing as well, and again with stimulation of either dominant or nondominant SMA. They suggest that stimulation of SMA may exert inhibitory effect on voluntary activity, not on speech exclusively but on motor activity as well. Talairach and Bancaud (1966) and Chauvel et al. (1985) found no left-right differences in the sites where speech arrest was elicited.

Similar to previous studies in humans (Penfield and Welch, 1951; Bates, 1953; Van Buren and Fedio, 1976; Woolsey et al., 1979; Morris et al., 1988), we have demonstrated some bilateral responses from unilateral stimulation of SMA. Nevertheless, the majority of the responses evoked with stimulation were contralateral. Bilateral body representation in SMA is often contrasted with the clearly contralateral representation in primary motor cortex. These findings are supported by animal studies demonstrating SMA neurons correlated with bilateral limb movements (Brinkman and Porter, 1979, 1983; Tanji and Kurata, 1981), although such cells have also been found in the primary motor area (Matsunami and Hamada, 1981). Patients with lesions in the SMA often exhibit a decrease in spontaneous motor activity bilaterally, although it is usually more pronounced contralaterally. Also, one of the few persistent deficits following SMA resection is a diminution in bimanual coordination (LaPlane et al., 1977; Freund, 1985; Freund and Hummelsheim, 1985). Therefore, it is conceivable that SMA plays a role in bilateral coordination of motor activity. We were, however, surprised to find that almost all of the bilateral and ipsilateral responses in our patients were obtained from right (nondominant) SMA stimulation. This is illustrated in the stimulation results in patient 8 (Fig. 2). Of the responses evoked at nine left (dominant) SMA sites (speech arrest excluded), all save one were contralateral, whereas all stimulation-evoked responses at five right SMA sites were bilateral. This raises the possibility that the right SMA exerts a more significant bilateral control than the left SMA. It could represent a right hemispheric dominance for bilateral initiation of motor acts, or bilateral motor "intention." Right cerebral dominance has been postulated for attentional and intentional mechanisms directed at the external milieu (Mesulam, 1981; Heilman et al., 1985; Meador et al., 1988). The attentional functions of the right hemisphere appear to span both hemispheres, while the left hemisphere seems to contain the neural apparatus mostly for contralateral attention (Mesulam, 1981). The left hemispace thus appears to be represented mostly by the right hemisphere, while the right hemispace is represented both by the ipsilateral right hemisphere and the contralateral left hemisphere. This may explain the findings that in patients with unilateral cerebral pathology, extinction of the left side of the body is more common than of the right side (Schwartz et al., 1979). The right supplementary motor cortex may be part of the right cerebral system dominant for attentional and intentional mechanisms directed at the body and the external space in which motor activity takes place. The hypothesis that the right SMA is more involved in bilateral motor control whereas the left SMA is involved primarily in control of the contralateral (right) side of the body suggests that the left side of the body may have less supplementary motor representation than the right side of the body, thus making it more prone to unilateral frontal lesions.

The responses obtained by stimulation of SMA have certain characteristic features that are often present in partial seizures thought to originate in SMA. These features include tonic posturing of one or more limbs, rhythmic movements of the extremities, forced turning of the head and eyes, vocalization, and speech arrest. These clinical characteristics may indicate recruiting of functional SMA regions near an active ictal focus (Penfield and Jasper, 1954; Green et al., 1980; Morris et al., 1988). Supplementary motor seizures are an exception to the rule that seizures involving all four extremities are accompanied by impairment of consciousness (Morris et al., 1988). This is explained by the fact that activation of one SMA is sufficient to elicit bilateral movements. It is unlikely that the responses obtained by electrical stimulation in this study represent a pathophysiology unique to patients with seizures. These responses were often obtained by stimulation in areas contralateral to or far away from a presumed focus or lesion and were not associated with epileptic discharges or stimulus afterdischarges. We also have not seen any consistent difference in the response pattern obtained in the patients with the structural lesions in this study compared to the patients without a radiologically apparent lesion.

In accordance with most animal studies, our study shows somatotopic organization in supplementary motor cortex with the upper extremities represented anterior to the lower extremities, and the face represented farther anteriorly. Eye movements were elicited in the same region where facial responses were evoked, and vocalization and speech arrest were evoked most anteriorly. At the posterior end of SMA, the transition from the primary motor representation of the foot area to supplementary motor representation of the lower extremity is not well defined, and we are not sure that the distinction between these two 
regions for the lower extremity can be made based on electrical stimulation mapping. The somatotopic electrical stimulation map of human SMA is in agreement with microstimulation data in animals (Gould et al., 1986; Mitz and Wise, 1987) and with the study in humans by Fox et al. (1985), who reported greater glucose metabolism (measured by positron emission tomography) in posterior SMA during hand movements, and greater metabolism farther anteriorly during eye movements. The failure to demonstrate SMA somatotopy with electrical stimulation in previous human studies is probably a result of poor stimulus control, especially in intraoperative studies, or an insufficient number of stimulation points or patients. Our study was done under favorable methodological conditions. Most patients had extensive medial sampling from both hemispheres. In some patients as many as 40 contacts were present. Also, good stimulus control was possible with gradual raising of stimulation currents under EEG monitoring to exclude afterdischarges. The opportunity to map extraoperatively reduced patient stress, improved ability to report sensory experiences, and enabled better behavioral control.

The human supplementary motor cortex emerging from this study is an area with divergent efferent output to the final motor pathways. Stimulation in this area coactivates larger groups of muscles than in the primary motor area, often in various body regions and bilaterally. Contrary to previous contentions based on studies of human SMA, we conclude that this area has somatotopical organization, although it may not be as precise as that of primary motor cortex. Taken together with the human SMA lesion data and other physiological studies using CBF and slow potential techniques, the subjective-sensory responses reported by our patients suggest that this arca is involved in the intention invested in motor activity. Additional data will be required to explore the hypothesis of more extensive bilateral motor control exerted by the right SMA.

\section{References}

Bates JA (1953) Stimulation of the medial surface of the human cerebral hemisphere after hemispherectomy. Brain 76:405-447.

Brickner RM (1940) A human cortical area producing repetitive phenomena when stimulated. J Neurophysiol 3:128-130.

Brinkman C, Porter R (1979) Supplementary motor area in the monkey. Activity of neurons during performance of a learned motor task. J Neurophysiol 42:681-709.

Brinkman C, Porter R (1983) Supplementary motor area and premotor area of monkey cerebral cortex: functional organization and activities of single neurons during performance of a learned movement. In: Motor control mechanisms in health and disease (Desmedt JE, ed), pp 393-420. New York: Raven.

Chauvel P, Bancaud J, Buser P (1985) Participation of the supplementary motor area in speech. Exp Brain Res 58:A14.

Deecke L (1985) Evidence from finger and toe movement-related potentials for SMA activation prior to human volitional acts. Exp Brain Res 58:A11-A12.

Deecke L (1987) Beritschaftpotentials as an indicator of movement preparation in supplementary motor area and motor cortex. In: Motor areas of the cerebral cortex. Ciba Foundation Symposium 132, pp 231-250. Chicester: Wiley.

Eccles JC (1982) The initiation of voluntary movements by supplementary motor area. Arch Psychiatr Nervenkr 231:423-441.

Eccles JC, Robinson DN (1984) The wonder of being human. Our brain and our mind. New York: Macmillan.

Erickson TC, Woolsey CN (1951) Observations on the supplementary motor area of man. Trans Am Neurol Assoc 76:50-56.

Foerster $O$ (1936) The motor cortex in man in the light of Hughlings Jackson's doctrines. Brain 59:135-159.

Fox PT, Fox JM, Raichle ME, Burde RM (1985) The role of cerebral cortex in the generation of voluntary saccades: a positron emission tomographic study. J Neurophysiol 2:348-369.

Freund HJ (1985) Motor disturbances after frontal lobe lesions in man. Exp Brain Res 598:A9-A10.

Freund HJ, Hummelsheim H (1985) Lesions of the premotor cortex in man. Brain 108:697-733.

Gould IIJ, Cusik CG, Pons TP, Kaas JH (1986) The relationship of corpus callosum connections to electrical stimulation maps of motor, supplementary motor, and frontal eyefields in the owl monkeys. J Comp Neurol 247:297-325.

Green JR, Angevine JB, White JC, Edes AD, Smith RD (1980) Significance of supplementary motor area in partial seizures and in cerebral localization. Neurosurgery 6:66-75.

Guidetti B (1957) Desordres de la parole associes a des lesions de la surface interhemispherique frontale posterieure. Rev Neurol (Paris) 97:121-131.

Heilman KM, Valenstein E, Watson RT (1985) The neglect syndrome. In: Handbook of clinical neurology, Vol 45, Clinical neuropsychology (Vinken PJ, Bruyn GW, Klawans HL, Frederis JAM, eds), pp 153184. Amsterdam: Elsevier.

Horsley V, Schafer EA (1888) A record of experiments upon the functions of the cerebral cortex. Philos Trans R Soc Lond [Biol] 179:145.

Hughes JR, Mazurowski JA (1962) Studies on the supracallosal mesial cortex of unanesthesized conscious mammals. II. Monkey. A. Movements elicited by electrical stimulation. Electroencephalogr Clin Neurophysiol 14:477-485.

Kubota K, Funahashi S (1982) Direction-specific activities of dorsolateral, prefrontal and motor cortex pyramidal tract neurons during visual tracking. J Neurophysiol 47:362-376.

LaPlane D, Talairach J, Meninger V, Bancaud J, Orgogozo JM (1977) Clinical consequences of corticectomies involving the supplementary motor area in man. J Neurol Sci 34:301-314.

Lucas JC, Requin J, Vitton N (1983) Anticipatory neuronal activity in the monkey precentral cortex during reaction time foreperiod: preliminary results. Exp Brain Res [Suppl] 7:120-127.

Macpherson JM, Marangoz C, Miles TS, Wiesendanger M (1982a) Microstimulation of the supplementary motor area (SMA) in the awake monkey. Exp Brain Res 45:410-416.

Macpherson JM, Wiesendanger M, Marangoz C, Miles TS (1982b) Corticospinal neurons in supplementary motor area of the monkey. A single unit study. Exp Brain Res 48:81-88.

Matsunami K, Hamada I (1981) Characteristics of the ipsilateral movement-related neurons in the motor cortex of the monkey. Brain Res 204:29-42.

Meador KJ, Loring DW, Lee GP, Brooks BS, Thompson EE, Thompson WO, Heilman KM (1988) Right cerebral specialization for tactile attention as evidenced by intracarotid sodium amytal. Neurology 38 : 1763-1766.

Mesulam MM (1981) A cortical network for directed attention and unilateral neglect. Ann Neurol 10:309-325.

Mitz AR, Wise SP (1987) The somatotopic organization of the supplementary motor area: intracortical microstimulation mapping. $J$ Neurosci 7:1010-1021.

Morris HH, Dinner DS, Luders H, Wyllie E, Kramer R (1988) Supplementary motor seizures: clinical and electroencephalographic findings. Neurology 38:1075-1082.

Munk H (1881) Uber der Functionen der Grosshirnrinde, Gasammelte Mitteilunger aus den Jahren 1877-1880, mit Einleitung under Anmerkunge. Berlin: Hirschwald.

Murray EA, Coulter JD (1981) Organization of corticospinal neurons in the monkey. J Comp Neurol 195:339-365.

Orgogozo JM, Larsen B (1979) Activation of the supplementary motor area during voluntary movement in man suggests it works as a supramotor area. Science 206:847-850.

Palmer C, Schmidt EM, McIntosh JS (1981) Corticospinal and corticorubral projections from the supplementary motor area in the monkey. Brain Res 209:305-314.

Penfield W, Jasper H (1954) Epilepsy and the functional anatomy of the human brain. Boston: Little, Brown.

Penfield W, Welch K (1951) The supplementary motor area of the cerebral cortex. A clinical and experimental study. Arch Neurol Psychiatry $66: 289-317$.

Roland PE, Larsen B, Lassen NA, Skinhoj E (1980) Supplementary motor area and other cortical areas in organization of voluntary movements in man. J Neurophysiol 43:118-136. 
Schwartz AS, Marchok PL, Kreinick CJ, Flynn RE (1979) The asymmetric lateralization of tactile extinction in patients with unilateral cerebral dysfunction. Brain 102:669-684.

Smith AM (1979) The activity of supplementary motor area neurons during a maintained precision grip. Brain Res 172:315-327.

Talairach J, Bancaud J (1966) The supplementary motor area in man. Int J Neurol 5:330-347.

Tanji J, Evarts EV (1976) Anticipatory activity of motor cortex neurons in relation to direction of an intended movement. J Neurophysiol 39:1062-1068.

Tanji J, Kurata K (1981) Contrasting neuronal activity in the ipsilateral and contralateral supplementary motor areas in relation to a movement of monkey's distal hindlimb. Brain Res 222:155-158.

Tanji J, Kurata K (1982) Comparison of movement-related neurons in two cortical motor areas in primates. $J$ Neurophysiol 40:633-653.

Van Buren JM, Fedio P (1976) Functional representation on the medial aspect of the frontal lobe in man. J Neurosurg 44:275-289.
Wiesendanger M, Hummelsheim H, Macpherson J (1985) Microelectrophysiology of the supplementary motor areas. Exp Brain Res 58: A2.

Wise SP, Tanji J (1981) Supplementary and precentral motor cortex: contrast in responsiveness to peripheral input to the hindlimb area of the unanesthesized monkey. J Comp Neurol 195:433-451.

Woolsey CN, Settlage PH, Meyer DR, Spencer W, Hamuy TP (1952) Patterns of localization in precentral and "supplementary" motor areas and their relation to the concept of premotor areas. Res Publ Assoc Nerv Ment Dis 30:238-264.

Woolsey CN, Erickson TC, Gilson WE (1979) Localization in somatic sensory and motor areas of human cerebral cortex as determined by direct evoked potentials and electrical stimulation. J Neurosurg 51: 476-506. 